\title{
Level attraction in a microwave optomechanical circuit
}

\author{
N. R. Bernier, L. D. Tóth, A. K. Feofanov, ${ }^{*}$ and T. J. Kippenberg ${ }^{\dagger}$ \\ Institute of Physics, École Polytechnique Fédérale de Lausanne (EPFL), CH-1015 Lausanne, Switzerland
}

(Received 25 September 2017; published 21 August 2018)

\begin{abstract}
Level repulsion - the opening of a gap between two degenerate modes due to coupling —is ubiquitous anywhere from solid-state theory to quantum chemistry. In contrast, if one mode has negative energy, the mode frequencies attract instead. They converge and develop imaginary components, leading to an instability; an exceptional point marks the transition. This only occurs if the dissipation rates of the two modes are comparable. Here we expose a theoretical framework for the general phenomenon and realize it experimentally through engineered dissipation in a multimode superconducting microwave optomechanical circuit. Level attraction is observed for a mechanical oscillator and a superconducting microwave cavity, while an auxiliary cavity is used for sideband cooling. Two exceptional points are demonstrated that could be exploited for their topological properties.
\end{abstract}

DOI: 10.1103/PhysRevA.98.023841

\section{INTRODUCTION}

Level repulsion of two coupled modes with an energy crossing has applications ranging from solid-state theory [1] to quantum chemistry [2]. While deceptively simple, it underpins more exotic phenomena. With the introduction of dissipation or gain, an exceptional point [3] appears that is topologically nontrivial [4-6]. The special case of two modes with equal dissipation and gain rates is an example of parity-time symmetry $[7,8]$. The spontaneous breaking of that symmetry is marked by the exceptional point. In recent years, exceptional points gathered significant interest and they were demonstrated in a variety of systems including active microwave circuits [9-11], lasers [12,13], and optical microresonators [14-16]. In particular, the topological transfer of energy between states by circling an exceptional point has been demonstrated with a microwave cavity [4], a microwave waveguide [17], as well as an optomechanical system [18,19].

Strikingly, if one mode has negative energy, the energy levels of two interacting modes do not repel, but attract instead [20-22]. The Hamiltonian leads to hybridized modes of complex eigenfrequencies, one of which is unstable. As in level repulsion, an exceptional point marks the transition between the regimes of real and complex frequencies. In the process, the real components of the frequencies become identical in a way that is reminiscent of the synchronization of driven oscillators [23].

Negative-energy modes (equivalent to harmonic oscillators with negative mass) have been studied in schemes to evade quantum measurement backaction [24-26]. Such a scheme was recently demonstrated with an atomic spin ensemble, prepared

\footnotetext{
*alexey.feofanov@epfl.ch

†tobias.kippenberg@epfl.ch
}

Published by the American Physical Society under the terms of the Creative Commons Attribution 4.0 International license. Further distribution of this work must maintain attribution to the author(s) and the published article's title, journal citation, and DOI. in its maximal-energy spin state in a magnetic field [27]. Spin flips decrease the energy and correspond to excitations of a harmonic oscillator with a negative mass. Alternatively, the negative-energy mode can be effectively realized in a frame rotating faster than the mode itself $[28,29]$.

In cavity optomechanics [30], a blue-detuned pump tone induces time-dependent interactions between the electromagnetic mode and the mechanical oscillator. In a frame rotating at the pump frequency, the Hamiltonian is time independent, and the electromagnetic mode appears to have negative energy. While level repulsion was demonstrated in the strong coupling regime of cavity optomechanics [31-33], level attraction has so far not been observed.

Here we construct a general theoretical framework for level attraction and demonstrate the phenomenon in a microwave optomechanical circuit using engineered dissipation. In Sec. II,
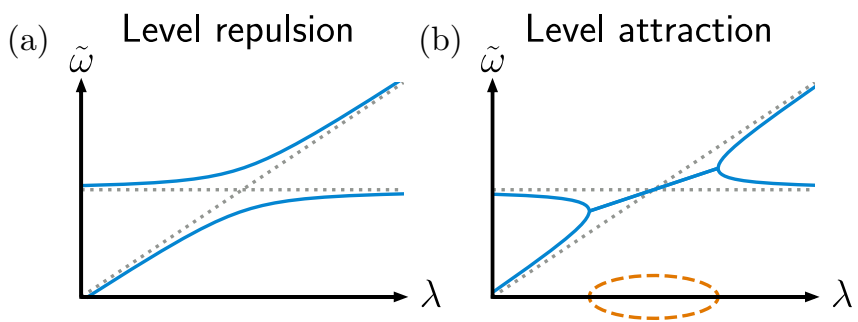

FIG. 1. Level repulsion and attraction. Two modes, whose bare frequencies depend on a parameter $\lambda$, have a level crossing (dotted lines). A coherent coupling will in general lift the degeneracy. (a) In the more usual case, level repulsion, the coupling opens a gap between the frequencies of the hybridized eigenmodes $\tilde{\omega}$ (blue solid lines) and the eigenfrequencies bend away from each other. (b) In contrast, if one of the modes has negative energy, level attraction occurs. The real components of the eigenfrequencies $\tilde{\omega}$ (blue solid lines) bend toward each other and converge. They meet at two exceptional points, where the curves have kinks. A gap opens in the imaginary components of the frequencies (orange dashed lines). The mode with a negative imaginary component to the frequency is unstable and grows exponentially. 
we show how a coherent coupling between modes of positive and negative energy gives rise to level attraction. The role of dissipation is discussed and explains the difficulty in observing level attraction in such systems, as the dissipation rates of the two modes must be similar. An intuitive way to classify different types of exceptional points in two-mode system is developed that allows us to clearly distinguish the cases of level repulsion and attraction. In Sec. III, both level attraction and repulsion are demonstrated experimentally in the same microwave optomechanical circuit, where the mechanical dissipation rate can be engineered to match that of the microwave cavity.

\section{THEORETICAL MODEL OF LEVEL ATTRACTION}

We start with a general theoretical model of a positiveenergy mode coherently coupled to a negative-energy one. The two modes, of annihilation operators $\hat{a}$ and $\hat{b}$ and coherently coupled with strength $g$, are described by the Hamiltonian

$$
\hat{H}=-\hbar \omega_{1}(\lambda) \hat{a}^{\dagger} \hat{a}+\hbar \omega_{2}(\lambda) \hat{b}^{\dagger} \hat{b}+\hbar g\left(\hat{a} \hat{b}+\hat{a}^{\dagger} \hat{b}^{\dagger}\right),
$$

where the two positive frequencies $\omega_{1}$ and $\omega_{2}$ vary with respect to an external parameter $\lambda$. The linear coupling chosen here is quite general: if we assume the modes close in frequency, the other linear terms $\hat{a}^{\dagger} \hat{b}$ and $\hat{a} \hat{b}^{\dagger}$ can be neglected in the rotating wave approximation (valid only if the frequencies $\omega_{1,2}$ dominate over the dissipation rates for an open system). The coupling rate $g$ is chosen to be real, because any complex phase can be absorbed in a redefinition of $\hat{a}$ or $\hat{b}$.

In the Heisenberg picture, this leads to the equations of motion

$$
\frac{d}{d t}\left(\begin{array}{c}
\hat{a} \\
\hat{b}^{\dagger}
\end{array}\right)=i\left(\begin{array}{cc}
\omega_{1} & -g \\
g & \omega_{2}
\end{array}\right)\left(\begin{array}{c}
\hat{a} \\
\hat{b}^{\dagger}
\end{array}\right),
$$

where we drop the explicit $\lambda$ dependence. We note that the uncoupled bare modes evolve as $\hat{a}(t)=e^{i \omega_{1} t} \hat{a}(0)$ and $\hat{b}^{\dagger}(t)=$ $e^{i \omega_{2} t} \hat{b}^{\dagger}(0)$ with a positive phase. The hybridized eigenmodes of the system are found by diagonalizing the matrix in Eq. (2), and have eigenfrequencies

$$
\tilde{\omega}_{1,2}=\frac{\omega_{1}+\omega_{2}}{2} \pm \sqrt{\left(\frac{\omega_{1}-\omega_{2}}{2}\right)^{2}-g^{2}} .
$$

The negative sign in front of $g^{2}$ is the only difference with the eigenfrequencies for the case of level repulsion (when $\hat{a}$ has positive energy) but it dramatically impacts the physics.

In Fig. 1, level attraction is compared to level repulsion, with two striking features. First, instead of avoiding each other, the eigenfrequencies pull toward one another. Second, when they meet at $4 g^{2}=\left(\omega_{1}-\omega_{2}\right)^{2}$, the frequencies acquire positive and negative imaginary parts, causing exponential decay and growth. The hybridized mode with a negative imaginary component grows exponentially and is therefore unstable. In Appendix A, level repulsion and attraction are compared more systematically.

The transition between the regimes of real and complex eigenfrequencies is marked by exceptional points, which can be understood by studying the matrix of Eq. (2). Decomposed in terms of Pauli matrices and omitting the term proportional to the identity, it can be expressed as $\frac{1}{2}\left(\omega_{1}-\omega_{2}\right) \sigma_{z}-i g \sigma_{y}$.
In contrast with level repulsion for which the interaction term would be $g \sigma_{x}$, here the Hermitian Pauli matrix is multiplied by an imaginary coefficient. If the first term has a larger amplitude, the eigenfrequencies are real, while they are complex if the second term dominates. When the two Pauli matrices have coefficients of the same amplitude, the matrix is proportional to $\sigma_{z}-i \sigma_{y}$. At this point, the two eigenvectors coalesce and a single eigenvector with a single eigenvalue subsists: it is an exceptional point [3]. More generally for all two-mode systems, any point for which the dynamics is determined by a matrix proportional to $\sigma_{\alpha}+i \sigma_{\beta}$, with $\alpha \neq \beta$, is an exceptional point. In Appendix B, we use this decomposition to construct an intuitive classification of the various realizations of exceptional points.

Level attraction arises whenever the coupling term consists of a Pauli matrix with an imaginary coefficient. In fact, coupled oscillators of positive and negative energy are only one way to achieve this. An alternative relies on a coupling between two modes that cannot be derived from a Hamiltonian, such as the dissipative interaction through one or multiple intermediary modes [34]. The mode hybridization observed between positive-energy oscillators with dissipative [18,35] and nonconservative [36] interactions can be interpreted as level attraction.

While level attraction of two linearly coupled modes displays intriguing similarities with the synchronization of driven oscillators, important differences exist. As in synchronization, the real components of the frequencies "lock" over a frequency range that increases with the coupling rate $g$, and form the equivalent of an Arnold tongue [23]. The physical process differs, however. In synchronization, one starts with two oscillators that are driven nonlinearly to their limit cycles, then (a)

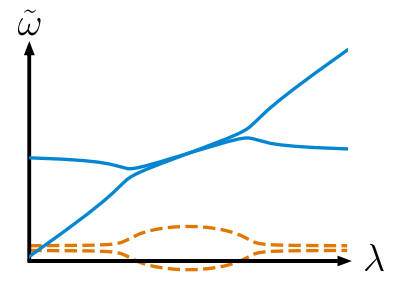

(b)

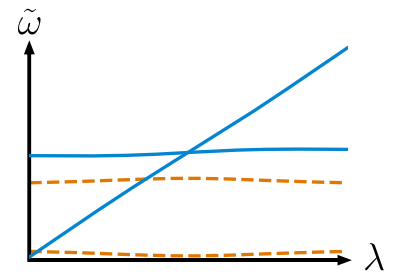

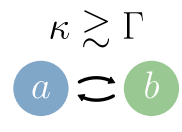

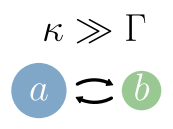

FIG. 2. Effect of dissipation on level attraction. Two modes, $\hat{a}$ and $\hat{b}$, cross in frequency (mode $\hat{a}$ having negative energy), with respective dissipation rates $\kappa$ and $\Gamma$. The real component of the frequency is the solid blue line and the imaginary component the dashed orange line. (a) While a finite average dissipation rate simply translates the imaginary components of the frequency, a difference in the two rates $(\kappa \neq \Gamma)$ affects qualitatively level attraction. The kinks and the exceptional points disappear and the picture is overall smoothed out. (b) When the dissipation rates differ significantly, no trace of level attraction is visible anymore. In both cases, one of the hybridized mode becomes unstable if the imaginary component of its frequency turns negative. 
a coupling is introduced that locks their frequencies and their phases [37]. In level attraction by contrast, the frequencies of the two modes attract through linear dynamics until they become identical. The state of the two hybridized modes remain independent and their phases can be set arbitrarily.

To understand why level attraction is in practice less common than level repulsion, the role of dissipation should be studied. We open the system and include in our treatment the energy dissipation rates $\kappa$ and $\Gamma$ respectively for the modes $\hat{a}$ and $\hat{b}$. They can be introduced as positive imaginary components of the bare frequencies in the equations of motion. The results of Eqs. (2) and (3) can be extended by replacing $\omega_{1}$ with $\omega_{1}+i \frac{\kappa}{2}$ and $\omega_{2}$ with $\omega_{2}+i \frac{\Gamma}{2}$. In Fig. 2, we compare the resulting eigenfrequencies. If the dissipation rates are equal $(\kappa=\Gamma)$, the level structure of Fig. 1(b) is reproduced with the imaginary components translated to a finite average. However, in the case of even slightly mismatched dissipation rates $\kappa \gtrsim \Gamma$ [Fig. 2(a)], the exceptional points and the kinks in the frequencies disappear. For increasingly dissimilar rates $\kappa \gg \Gamma$ [Fig. 2(b)], the level-attraction picture progressively disappears until the modes seem to cross without interacting. Therefore, only in a system where dissipation rates can be tuned to closely match each other is level attraction observable.

\section{DEMONSTRATION IN A MICROWAVE OPTOMECHANICAL CIRCUIT}

Cavity optomechanics provides an ideal setting to study level attraction and compare it to level repulsion. We now take $\hat{a}$ to represent an electromagnetic mode and $\hat{b}$ a mechanical oscillator, coupled through the optomechanical interaction $\hbar g_{0} \hat{a}^{\dagger} \hat{a}\left(\hat{b}+\hat{b}^{\dagger}\right)$, where $g_{0}$ is the vacuum optomechanical coupling [30]. With a blue-detuned pump tone applied to the system, the three-wave-mixing coupling is linearized and the Hamiltonian reduces to the form of Eq. (1)

$$
\hat{H}=-\hbar \Delta \hat{a}^{\dagger} \hat{a}+\hbar \Omega_{\mathrm{m}} \hat{b}^{\dagger} \hat{b}+\hbar g\left(\hat{a} \hat{b}+\hat{a}^{\dagger} \hat{b}^{\dagger}\right),
$$

where $\Delta$ is the detuning of the pump tone, $\Omega_{\mathrm{m}}$ the mechanical mode frequency, and $g=g_{0} \sqrt{n_{\mathrm{c}}}$ the linear coupling enhanced by the mean cavity photon number $n_{\mathrm{c}}$ due to the pump tone. As above, we neglect counter-rotating terms and assume the detuning $\Delta$ to be close to $\Omega_{\mathrm{m}}$. Critically, the Hamiltonian is expressed in a frame rotating at the pump frequency in order to be time independent. Hence, for a blue detuning $\Delta>0$, the cavity mode effectively has a negative energy, since the photons have a negative relative frequency with respect to the pump. In this context, the well-known parametric instability of optomechanics [30] can be interpreted as resulting from the physics of level attraction. The instability stems from the negative imaginary component that develops in the eigenfrequencies of the equations of motion, above the critical coupling $g_{\text {crit }}=$ $\sqrt{\kappa \Gamma} / 2$. For level attraction to be observable, the magnitudes of $\kappa$ and $\Gamma$ should be close. For the usual experimental parameters, however, the electromagnetic decay rate $\kappa$ is much larger than the mechanical rate $\Gamma$, and no attraction can in practice be observed for the mechanical and electromagnetic modes.

In our experiment, the effective mechanical energy decay rate $\Gamma_{\text {eff }}$ is artificially increased to match $\kappa$ using sideband cooling with an auxiliary mode. We use a superconducting electromechanical circuit [32] containing two microwave

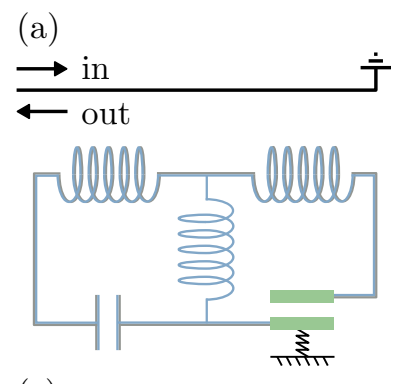

(c)

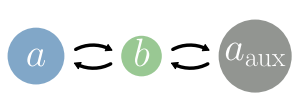

(d)

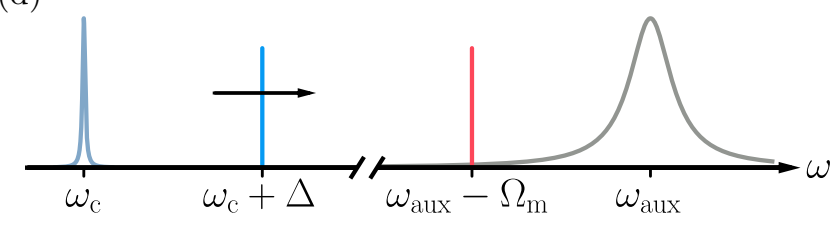

FIG. 3. Engineering dissipation in a multimode optomechanical circuit. To observe level attraction, the dissipation rate $\Gamma$ of the mechanical mode $\hat{b}$ must be increased to match $\kappa$, the much larger dissipation rate of the primary electromagnetic mode $\hat{a}$. To that end, an auxiliary mode $\hat{a}_{\text {aux }}$ is used for sideband cooling. (a) Schematic of the microwave optomechanical circuit, coupled inductively to a microwave feedline and measured in reflection. The two hybridized modes of the circuit, $\hat{a}$ and $\hat{a}_{\text {aux }}$, interact with the motion of the top membrane of a shared capacitor, acting as the mechanical oscillator $\hat{b}$ (in green). (b) Photograph of the circuit and scanning-electron micrograph of the vacuum-gap capacitor. (c) Diagram of the three interacting modes. (d) Frequency domain representation of the levelattraction experiment. A microwave pump tone (vertical red line), red detuned by the mechanical frequency $\Omega_{\mathrm{m}}$ with respect to the auxiliary mode resonance $\omega_{\text {aux }}$ (grey peak) is used for sideband cooling. Level attraction of the modes $\hat{a}$ and $\hat{b}$ is achieved by sweeping the detuning $\Delta$ of a pump tone (vertical blue line) near the blue sideband of the primary mode resonance $\omega_{\mathrm{c}}$ (blue peak). For level repulsion, the pump tone is instead swept near the red sideband.

modes interacting with the vibrational mode of a vacuum-gap capacitor [represented schematically in Fig. 3(a) and shown in Fig. 3(b)]. The design, which was demonstrated in previous work [38], uses two hybridized electromagnetic modes of the circuit to ensure that one has a much larger external coupling rate to the microwave feedline than the other. The more dissipative auxiliary mode $\hat{a}_{\text {aux }}$ is used to perform sideband cooling of the mechanical oscillator with a red-detuned pump tone. This damps the oscillator and increases its effective dissipation rate to $\Gamma_{\text {eff }} \approx \kappa$. Meanwhile, the less dissipative primary mode $\hat{a}$ undergoes level attraction with the damped mechanical oscillator.

In the experiment, the device is placed inside a dilution refrigerator and cooled to the base temperature, below $50 \mathrm{mK}$, at which the circuit is superconducting and its internal $Q$ factor is enhanced. The two microwave modes $\hat{a}$ and $\hat{a}_{\text {aux }}$ have respective resonance frequencies $\omega_{\mathrm{c}} \approx 2 \pi \times 4.11 \mathrm{GHz}$ and $\omega_{\text {aux }} \approx 2 \pi \times 5.22 \mathrm{GHz}$, and dissipation rates $\kappa \approx 2 \pi \times 110 \mathrm{kHz}$ and $\kappa_{\mathrm{aux}} \approx 2 \pi \times 1.8 \mathrm{MHz}$. They interact with the fundamental vibrational mode of the top plate of the vacuum-gap capacitor, which has a frequency $\Omega_{\mathrm{m}} \approx 2 \pi \times 6.3 \mathrm{MHz}$. By placing a pump 

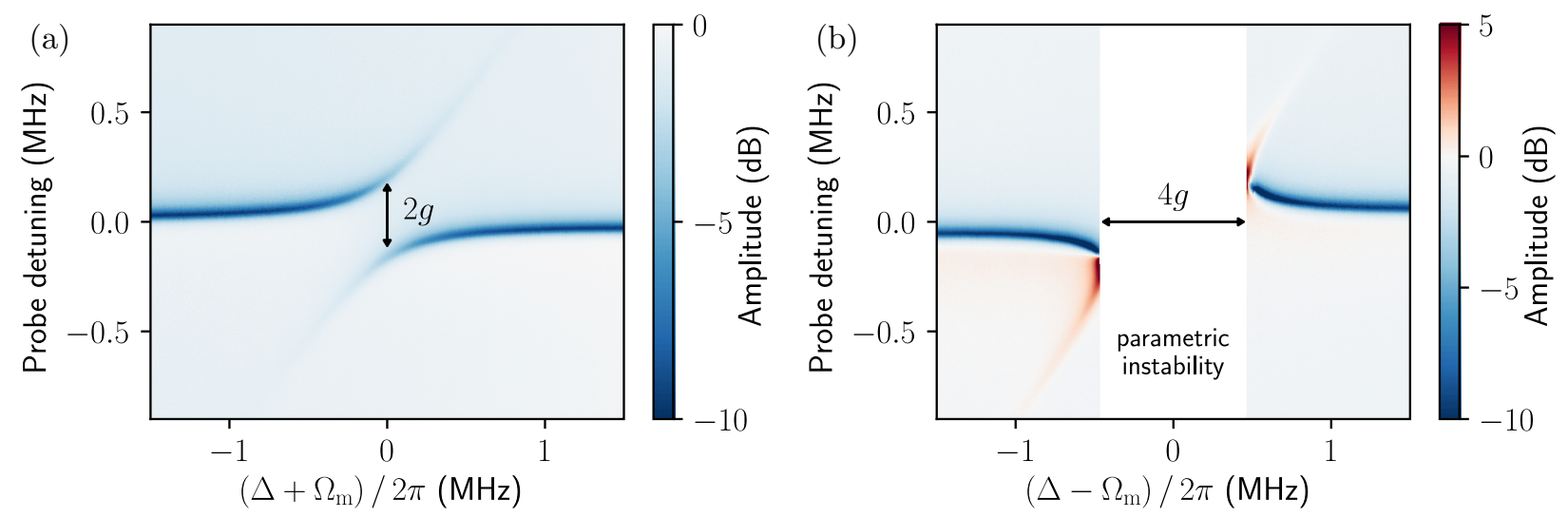

FIG. 4. Experimental demonstration of level repulsion and attraction in a microwave optomechanical circuit. Amplitude response of the system as the detuning $\Delta$ of the pump tone is varied, when the effective dissipation rate of the mechanical mode $\hat{b}$ matches that of the microwave mode $\hat{a}$. In the laboratory frame, $\Delta$ determines the effective frequency of the mechanical oscillator, which is swept across the microwave resonance. (a) When the pump tone is swept in frequency across the red sideband of the microwave mode, the two resonances bend away from each other. (b) If the pump tone is swept across the blue sideband instead, the resonances pull toward each other and converge near two exceptional points. Data are omitted for clarity where the system becomes unstable, and one hybridized mode grows exponentially until the conditions of validity of Eq. (4) are no longer fulfilled (parametric instability).

tone that is red detuned by $\Omega_{\mathrm{m}}$ from the auxiliary mode resonance [see Fig. 3(d)], the mechanical oscillator is damped. The mechanical dissipation rate $\Gamma$, originally below $2 \pi \times 100 \mathrm{~Hz}$, is tuned to an effective dissipation rate $\Gamma_{\text {eff }} \approx \kappa \approx 2 \pi \times 110 \mathrm{kHz}$.

Level repulsion and attraction of the primary microwave mode and the damped mechanical oscillator are both measured. As a pump tone is tuned to the blue or red sideband of the primary microwave mode [the former case is illustrated in Fig. 3(d)], the weak probe tone of a vector network analyzer is applied near the resonance of the microwave cavity to obtain its linear response. Due to the hybridization of the modes, the response carries information about both microwave and mechanical modes. For both red and blue detunings, the same pump power is set to obtain a coupling strength $g \approx$ $2 \pi \times 200 \mathrm{kHz}$ corresponding to a mean cavity photon number $n_{\mathrm{c}} \approx 4 \times 10^{6}$.

The known case of level repulsion is obtained with a reddetuned tone [Fig. 4(a)]. As the bare effective mechanical mode frequency comes near the microwave resonance, the two modes hybridize; their eigenfrequencies bend away from each other with a gap of $2 g$.

With a blue-detuned tone, level attraction occurs instead. The response, shown in Fig. 4(b), displays the characteristic level structure of Fig. 1(b). The resonance frequencies of the modes attract and converge to the points where the bare frequencies of the modes differ only by $\pm 2 g$. In order for the level attraction to be clearly visible, the coupling rate $g$ is set to dominate over the dissipation rates $\kappa$ and $\Gamma_{\text {eff }}$. It therefore exceeds the critical coupling $g_{\text {crit }}$, resulting in parametric instability. One of the modes grows exponentially until the conditions of the validity of Eq. (4) are no longer fulfilled. Namely, the fluctuating field is no longer negligible compared to the mean cavity photon number $n_{\mathrm{c}}$. The original nonlinear optomechanical interaction constrains the system to a limit cycle with a modified cavity resonance frequency [39], the description of which lies beyond the scope of this article. Data are omitted for clarity in the unstable region.

\section{CONCLUSION}

In summary, level attraction was experimentally demonstrated using a dual-mode electromechanical circuit. Although related to the well-studied parametric instability of optomechanics, the vastly different dissipation rates for the mechanical and electromagnetic modes prevented its observation until now. Level attraction, similar to level repulsion in open systems, gives rise to exceptional points. In both cases, the real part of the frequencies converge and a gap opens in the imaginary part (or vice versa) precisely at the exceptional point. In future work, the exceptional points of level attraction could be harnessed to demonstrate topological phenomena by circling such a point in a two-dimensional parameter space $[4,17,18]$. Since the exceptional point only exists when the dissipation rates of the two modes match exactly, the tunable mechanical damping rate $\Gamma_{\text {eff }}$ can be used as one parameter in such an experiment, with the tunable coupling rate $g$ as the second.

The data and code used to produce the figures of this work are available online [40].

\section{ACKNOWLEDGMENTS}

We thank A. Nunnenkamp and D. Malz for useful discussions and comments. This work was supported by the Swiss National Funds (SNF) (Grant No. 163387), the NCCR Quantum Science and Technology (QSIT) (Grant No. 51NF40160591), and the EU Horizon 2020 research and innovation program under Grant Agreement No. 732894 (FET Proactive HOT). T.J.K. acknowledges financial support from an ERC AdG (QuREM) (Grant No. 320966). All samples were fabricated in the Center of MicroNanoTechnology (CMi) at EPFL.

\section{APPENDIX A: SYMMETRY BETWEEN LEVEL REPULSION AND ATTRACTION}

We explicitly derive here two minimal models, one for the usual level repulsion of two coherently coupled modes, the 
other for level attraction of a negative-energy mode coherently coupled to a positive-energy mode. We show that a symmetry relation links the two cases. They are images of each other under the exchange of frequency and dissipation rates.

First, we review level repulsion. Two modes of positive energy (with annihilation operators $\hat{a}$ and $\hat{b}$, and respective frequencies $\omega_{1}$ and $\omega_{2}$ ) interact, as described by the Hamiltonian

$$
\hat{H}_{\mathrm{LR}}=\hbar \omega_{1} \hat{a}^{\dagger} \hat{a}+\hbar \omega_{2} \hat{b}^{\dagger} \hat{b}+\hbar g\left(\hat{a} \hat{b}^{\dagger}+\hat{a}^{\dagger} \hat{b}\right),
$$

with $g$ the linear coupling strength. The equation of motion in the Heisenberg picture is given by

$$
\frac{d}{d t}\left(\begin{array}{c}
\hat{a} \\
\hat{b}
\end{array}\right)=-i\left(\begin{array}{cc}
\omega_{1} & g \\
g & \omega_{2}
\end{array}\right)\left(\begin{array}{l}
\hat{a} \\
\hat{b}
\end{array}\right) .
$$

To solve the system in terms of eigenmodes, the matrix is diagonalized. The eigenfrequencies are given by

$$
\tilde{\omega}_{1,2}^{\mathrm{LR}}=\frac{\omega_{1}+\omega_{2}}{2} \pm \sqrt{\left(\frac{\omega_{1}-\omega_{2}}{2}\right)^{2}+g^{2}} .
$$

The model is extended to describe modes with dissipation by adding imaginary components to the bare frequencies, substituting $\omega_{1} \rightarrow \omega_{1}-i \Gamma_{1} / 2$ and $\omega_{2} \rightarrow \omega_{2}-i \Gamma_{2} / 2$. Note that a negative sign is required to obtain decaying exponentials. For ease of notation, we define $\omega_{0}=\frac{\omega_{1}+\omega_{2}}{2}, \Gamma_{0}=\frac{\Gamma_{1}+\Gamma_{2}}{2}$, $\Delta \omega=\omega_{1}-\omega_{2}$, and $\Delta \Gamma=\Gamma_{1}-\Gamma_{2}$. The eigenfrequencies can now be written as

$$
\tilde{\omega}_{1,2}^{\mathrm{LR}}=\omega_{0}-i \frac{\Gamma_{0}}{2} \pm \sqrt{\left(\frac{\Delta \omega-i \Delta \Gamma / 2}{2}\right)^{2}+g^{2}} .
$$

The frequency of oscillation of the eigenmodes is given by the real component of $\tilde{\omega}_{1,2}^{\mathrm{LR}}$ and the dissipation rate by half its imaginary component (with a minus sign).

We now consider level attraction. One (and only one) mode has negative energy such that the system is described by the Hamiltonian

$$
\hat{H}_{\mathrm{LA}}=-\hbar \omega_{1} \hat{a}^{\dagger} \hat{a}+\hbar \omega_{2} \hat{b}^{\dagger} \hat{b}+\hbar g\left(\hat{a} \hat{b}+\hat{a}^{\dagger} \hat{b}^{\dagger}\right)
$$

The equations of motion are given by

$$
\left(\begin{array}{c}
\hat{a} \\
\hat{b}^{\dagger}
\end{array}\right)=i\left(\begin{array}{cc}
\omega_{1} & -g \\
g & \omega_{2}
\end{array}\right)\left(\begin{array}{c}
\hat{a} \\
\hat{b}^{\dagger}
\end{array}\right)
$$

with eigenfrequencies

$$
\tilde{\omega}_{1,2}^{\mathrm{LA}}=\frac{\omega_{1}+\omega_{2}}{2} \pm \sqrt{\left(\frac{\omega_{1}-\omega_{2}}{2}\right)^{2}-g^{2}} .
$$

The only difference with Eq. (A3) is the sign in front of $g^{2}$, which can result in a complex eigenvalue, meaning an instability for the system.

It might not be intuitive that the Hermitian Hamiltonian in Eq. (A5) leads to complex eigenfrequencies and unstable dynamics. In fact, in an infinite-dimensional Hilbert space, an operator must be compact as well as Hermitian to guarantee the existence of real eigenvalues [41], which is not the case here. We also note that only when the eigenfrequencies are real can the eigenoperators be interpreted as Bogoliubov modes [42]. When the eigenfrequencies are complex, the required commutation relations cannot be satisfied.
To include dissipation, we substitute again $\omega_{1} \rightarrow \omega_{1}+$ $i \Gamma_{1} / 2$ and $\omega_{2} \rightarrow \omega_{2}+i \Gamma_{2} / 2$. Note that the opposite sign for the imaginary component is required here to obtain decaying exponentials. The eigenfrequencies including dissipation become

$$
\tilde{\omega}_{1,2}^{\mathrm{LA}}=\omega_{0}+i \Gamma_{0} / 2 \pm \sqrt{\left(\frac{\Delta \omega+i \Delta \Gamma / 2}{2}\right)^{2}-g^{2}} .
$$

There is a symmetry between Eqs. (A4) and (A8). They are equivalent under the transformation $\omega^{\prime}=\Gamma / 2$ and $\Gamma^{\prime}=2 \omega$, if Eq. (A8) is multiplied by a factor $-i$ :

$$
\tilde{\omega}_{1,2}^{\mathrm{LA}}=\omega_{0}^{\prime}-i \Gamma_{0}^{\prime} / 2 \pm \sqrt{\left(\frac{\Delta \omega^{\prime}-i \Delta \Gamma^{\prime} / 2}{2}\right)^{2}+g^{2}} .
$$

We conclude that level attraction and repulsion are equivalent to each other through the exchange of frequency and dissipation rates (within a factor of 2). In Fig. 5, this
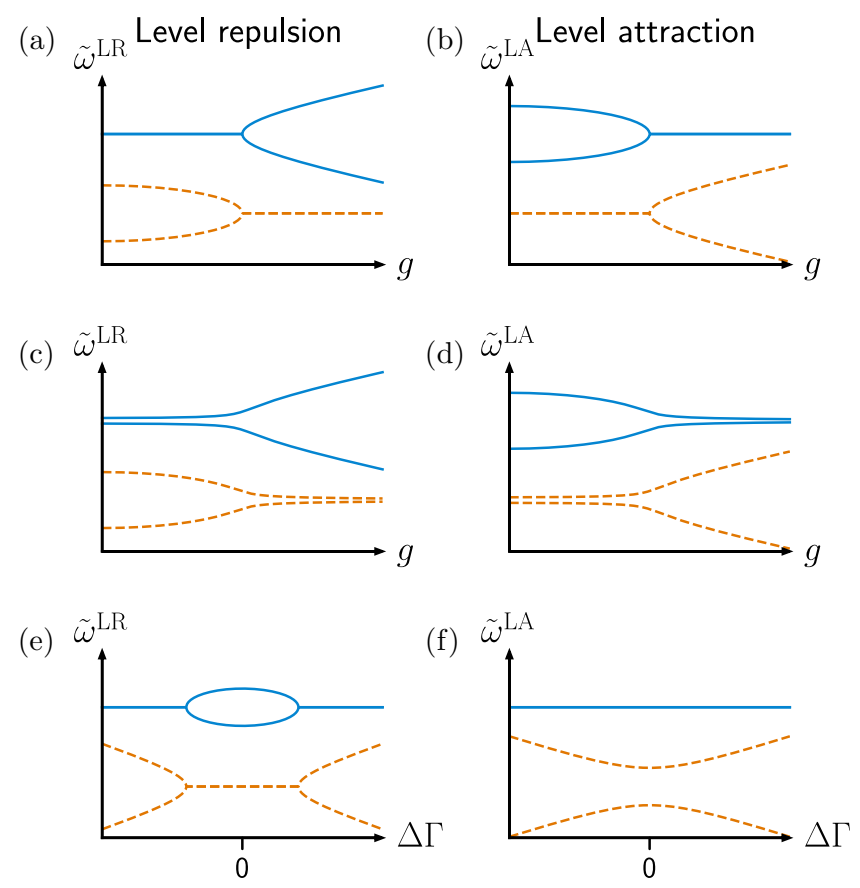

FIG. 5. Comparison of the real (solid blue lines) and imaginary (dashed orange lines) components of the eigenfrequencies $\tilde{\omega}$ for the level-repulsion Hamiltonian $\hat{H}_{\mathrm{LR}}$ (left column) and the level-attraction Hamiltonian $\hat{H}_{\text {LA }}$ (right column). (a),(b) The transition through an exceptional point, as a function of the coupling strength $g$. For level repulsion (a), the modes are originally degenerate $(\Delta \omega=0)$, while for level attraction (b) they originally have matching dissipation rates $(\Delta \Gamma=0)$. (c),(d) Same as (a),(b) with a slight nondegeneracy [ $\Delta \omega \neq 0$ for level repulsion (c) and $\Delta \Gamma \neq 0$ for level attraction (d)], such that the exceptional point is avoided. (e),(f) The difference in dissipation rates $\Delta \Gamma$ is varied, for degenerate modes $\Delta \omega=0$. This is the complementary case to Fig. 1 , where $\Delta \Gamma=0$ and $\Delta \omega$ is varied. For level repulsion (e), the imaginary component of the frequency attract and converge in a region where a gap opens in the real components of the frequency (analogous to level attraction as a function of $\Delta \omega$ ). For level attraction (f), the real component is unchanged while the dissipation rates have an avoided crossing (analogous to level repulsion as a function of $\Delta \omega$ ). 
symmetry is highlighted by contrasting equivalent situations for both level repulsion and level attraction Hamiltonians. In Figs. 5(a)-5(d), the curves for the real and imaginary parts of the eigenfrequencies are interchanged when going from the left column (level repulsion) to the right (level attraction). Figures 5(e) and 5(f) can be compared with Fig. 1 of the main text, where the characteristic shape of level attraction is here seen for the dissipation rates for the Hamiltonian of level repulsion and vice versa. In general, for level repulsion, when a coupling is introduced the frequencies repel, while the dissipation rates attract. The exact opposite is true for level attraction: a coupling makes the dissipation rates repel and the frequencies attract.

\section{APPENDIX B: CLASSIFICATION OF EXCEPTIONAL POINTS FOR SYSTEMS OF TWO COUPLED MODES}

We describe here how to classify exceptional points of $2 \times 2$ matrices using Pauli matrices. The classification is then used to sort recent experimental demonstrations of exceptional points.

In general, the equations of motion for a two-mode system can be written in the form

$$
i \frac{d}{d t}\left(\begin{array}{l}
\hat{d}_{1} \\
\hat{d}_{2}
\end{array}\right)=M\left(\begin{array}{l}
\hat{d}_{1} \\
\hat{d}_{2}
\end{array}\right)
$$

where $\hat{d}_{1}, \hat{d}_{2}$ are operators for the two modes and $M$ is a $2 \times 2$ matrix. The eigenmodes of the system and their eigenfrequencies correspond to the eigenvectors and eigenvalues of $M$. If for some parameter values, $M$ only has a single eigenvector and a single eigenvalue, this is called an exceptional point.

The matrix $M$ can always be decomposed in terms of the Pauli matrices, defined as

$$
\sigma_{x}=\left(\begin{array}{ll}
0 & 1 \\
1 & 0
\end{array}\right), \quad \sigma_{y}=\left(\begin{array}{cc}
0 & -i \\
i & 0
\end{array}\right), \quad \text { and } \quad \sigma_{z}=\left(\begin{array}{cc}
1 & 0 \\
0 & -1
\end{array}\right),
$$

in the form

$$
M=a_{0} \mathbb{1}+a_{1} \sigma_{x}+a_{2} \sigma_{y}+a_{3} \sigma_{z}
$$

with $\mathbb{1}$ the identity matrix and $a_{i}$ complex numbers. The eigenvalues of $M$ are now easily expressed as

$$
\lambda_{1,2}=a_{0} \pm \sqrt{a_{1}^{2}+a_{2}^{2}+a_{3}^{2}} .
$$

Note that the first term in Eq. (B3), proportional to the identity, only shifts the eigenvalues by a constant and has no effect on the eigenvectors. Anytime that the matrix $M$ can be written as a multiple of $\sigma_{\alpha}+i \sigma_{\beta}(\alpha \neq \beta)$ (plus the identity), there is only one eigenvalue and this is an exceptional point [43]. We can use this decomposition to classify examples of exceptional points.

(I) The most common case is the level repulsion of two (positive-energy) modes of degenerate frequencies due to a coherent coupling. Many experimentally demonstrated exceptional points fall in this category $[4,10-15,17]$. The two modes are $\hat{d}_{1}=\hat{a}$ and $\hat{d}_{2}=\hat{b}$, in our previous notation. The matrix $M$ can be written as

$$
M=\left(\omega_{0}-i \frac{\Gamma_{0}}{2}\right) \mathbb{1}-i \frac{\Delta \Gamma}{4} \sigma_{z}+g \sigma_{x} .
$$

For low coupling rate $g, M$ is diagonal with a gap for dissipation rates. For large $g$, the last term dominates such that the eigenmodes are eigenvectors of $\sigma_{x}: g$ opens a gap in frequency, while the dissipation rates are evenly distributed between the modes. The transition between the regimes is marked by an exceptional point at $g=\Delta \Gamma / 4$.

(II) In this article, we consider the case of level attraction of modes of negative and positive energies and matching dissipation rates. Here the operators are $\hat{d}_{1}=\hat{a}$ and $\hat{d}_{2}=\hat{b}^{\dagger}$ in our notation. The matrix $M$ can be written as

$$
-M=\left(\omega_{0}+i \frac{\Gamma_{0}}{2}\right) \mathbb{1}+\frac{\Delta \omega}{2} \sigma_{z}-i g \sigma_{y} .
$$

At low coupling $g$, there is a gap in frequency, while at high coupling, the $\sigma_{y}$ term opens a gap in dissipation rates and the frequencies are identical. An exceptional point marks the transition at $g=\Delta \omega / 2$. Note that the coherent coupling corresponds to a term with an imaginary coefficient for a system with one mode of negative energy.

(III) Level attraction of two modes can be realized in any system in which the coupling term has an imaginary component. In particular, dissipative interactions [34] represent an alternative way to the one presented in this article. The matrix $M$ is there expressed as

$$
M=\left(\omega_{0}-i \frac{\Gamma_{\mathrm{eff}}}{2}\right) \mathbb{1}+\frac{\Delta \omega}{2} \sigma_{z}+i g_{\mathrm{dis}} \sigma_{x},
$$

where for simplification the effective dissipation rate $\Gamma_{\text {eff }}$ of the two coupled modes is taken to be approximately equal. The effective interaction between the two modes has an imaginary coefficient and they have an increased effective dissipation rate $\Gamma_{\text {eff }}$ due to the dissipative interaction as well. Similar to the previous case, by increasing the coupling $g_{\text {dis }}$ the original gap in frequency is closed and a difference in dissipation rates is created. In contrast, however, $\Gamma_{\text {eff }}$ grows proportionally with $g_{\text {dis }}$, such that the gap in dissipation rates does not result in an instability. The mode hybridization between modes of positive energy coupled with dissipative interactions can be interpreted as level attraction.

Examples of this type of exceptional points were realized experimentally. In the experiment of $\mathrm{Xu}$ et al. [18], two mechanical oscillators are effectively coupled by both interacting with the same optical cavity. In the experiment of Khanbeykyan et al. [35], two modes of an optical resonator interact through multiple quantum dots. In the experiment of Gloppe et al. [36], two modes of a nanowire interact through the nonconservative force of an optical field, which although not dissipative in nature, cannot be derived from a Hamiltonian in a similar way.

(IV) Finally, yet another way to implement an exceptional point was realized in the experiment of Chen et al. [16]. The clockwise and counterclockwise modes of a whisperinggallery-mode resonator (of degenerate frequencies and dissipation rates) interact through two Rayleigh scatterers. This results in a combination of coherent and dissipative interaction that can be described by

$$
M=\left(\omega_{0}-i \frac{\Gamma_{0}}{2}\right) \mathbb{1}+g_{\mathrm{coh}} \sigma_{x}+i g_{\mathrm{dis}} \sigma_{y} .
$$

As the coupling $g_{\text {coh }}$ and $g_{\text {dis }}$ are varied, the relative phases of the bare modes that compose the hybrid eigenmodes change. When the two coupling strengths match $\left(g_{\text {coh }}=g_{\text {dis }}\right)$, the two 
eigenmodes coalesce and there is an exceptional point. Interestingly, this corresponds to a point of maximal nonreciprocity, with one bare mode coupled to the second but not the other way around [44].
[1] N. Ashcroft and N. Mermin, Solid State Physics (Brooks Cole, New York, 1976).

[2] P. Atkins and J. de Paula, Atkins' Physical Chemistry, 9th ed. (Oxford University Press, Oxford, 2009).

[3] W. D. Heiss, J. Phys. A 45, 444016 (2012).

[4] C. Dembowski, H.-D. Gräf, H. L. Harney, A. Heine, W. D. Heiss, H. Rehfeld, and A. Richter, Phys. Rev. Lett. 86, 787 (2001).

[5] R. Uzdin, A. Mailybaev, and N. Moiseyev, J. Phys. A 44, 435302 (2011).

[6] T. J. Milburn, J. Doppler, C. A. Holmes, S. Portolan, S. Rotter, and P. Rabl, Phys. Rev. A 92, 052124 (2015).

[7] C. M. Bender, Contemp. Phys. 46, 277 (2005).

[8] C. M. Bender, B. K. Berntson, D. Parker, and E. Samuel, Am. J. Phys. 81, 173 (2013).

[9] T. Stehmann, W. D. Heiss, and F. G. Scholtz, J. Phys. A 37, 7813 (2004).

[10] J. Schindler, A. Li, M. C. Zheng, F. M. Ellis, and T. Kottos, Phys. Rev. A 84, 040101 (2011).

[11] S. Assawaworrarit, X. Yu, and S. Fan, Nature 546, 387 (2017).

[12] M. Brandstetter, M. Liertzer, C. Deutsch, P. Klang, J. Schöberl, H. E. Türeci, G. Strasser, K. Unterrainer, and S. Rotter, Nat. Commun. 5, 4034 (2014).

[13] B. Peng, Ş. K. Özdemir, S. Rotter, H. Yilmaz, M. Liertzer, F. Monifi, C. M. Bender, F. Nori, and L. Yang, Science 346, 328 (2014).

[14] C. M. Bender, M. Gianfreda, Ş. K. Özdemir, B. Peng, and L. Yang, Phys. Rev. A 88, 062111 (2013).

[15] B. Peng, Ş. K. Özdemir, F. Lei, F. Monifi, M. Gianfreda, G. L. Long, S. Fan, F. Nori, C. M. Bender, and L. Yang, Nat. Phys. 10, 394 (2014).

[16] W. Chen, Ş. Kaya Özdemir, G. Zhao, J. Wiersig, and L. Yang, Nature 548, 192 (2017).

[17] J. Doppler, A. A. Mailybaev, J. Böhm, U. Kuhl, A. Girschik, F. Libisch, T. J. Milburn, P. Rabl, N. Moiseyev, and S. Rotter, Nature 537, 76 (2016).

[18] H. Xu, D. Mason, L. Jiang, and J. G. E. Harris, Nature 537, 80 (2016).

[19] H. Jing, S. K. Özdemir, X.-Y. Lü, J. Zhang, L. Yang, and F. Nori, Phys. Rev. Lett. 113, 053604 (2014).

[20] N. R. Bernier, E. G. Dalla Torre, and E. Demler, Phys. Rev. Lett. 113, 065303 (2014).

[21] H. Eleuch and I. Rotter, Acta Polytech. 54, 106 (2014).

[22] A. P. Seyranian, O. N. Kirillov, and A. A. Mailybaev, J. Phys. A 38, 1723 (2005).
[23] A. Pikovsky, M. Rosenblum, and J. Kurths, Synchronization: A Universal Concept in Nonlinear Sciences (Cambridge University Press, New York, 2003).

[24] M. Tsang and C. M. Caves, Phys. Rev. X 2, 031016 (2012).

[25] K. Zhang, P. Meystre, and W. Zhang, Phys. Rev. A 88, 043632 (2013).

[26] E. S. Polzik and K. Hammerer, Ann. Phys. (NY) 527, A15 (2015).

[27] C. B. Møller, R. A. Thomas, G. Vasilakis, E. Zeuthen, Y. Tsaturyan, M. Balabas, K. Jensen, A. Schliesser, K. Hammerer, and E. S. Polzik, Nature 547, 191 (2017).

[28] M. J. Woolley and A. A. Clerk, Phys. Rev. A 87, 063846 (2013).

[29] C. F. Ockeloen-Korppi, E. Damskägg, J.-M. Pirkkalainen, A. A. Clerk, M. J. Woolley, and M. A. Sillanpää, Phys. Rev. Lett. 117, 140401 (2016).

[30] M. Aspelmeyer, T. J. Kippenberg, and F. Marquardt, Rev. Mod. Phys. 86, 1391 (2014).

[31] S. Gröblacher, K. Hammerer, M. R. Vanner, and M. Aspelmeyer, Nature 460, 724 (2009).

[32] J. D. Teufel, D. Li, M. S. Allman, K. Cicak, A. J. Sirois, J. D. Whittaker, and R. W. Simmonds, Nature 471, 204 (2011).

[33] E. Verhagen, S. Deléglise, S. Weis, A. Schliesser, and T. J. Kippenberg, Nature 482, 63 (2012).

[34] A. Metelmann and A. A. Clerk, Phys. Rev. Lett. 112, 133904 (2014).

[35] M. Khanbekyan, H. A. M. Leymann, C. Hopfmann, A. Foerster, C. Schneider, S. Höfling, M. Kamp, J. Wiersig, and S. Reitzenstein, Phys. Rev. A 91, 043840 (2015).

[36] A. Gloppe, P. Verlot, E. Dupont-Ferrier, A. Siria, P. Poncharal, G. Bachelier, P. Vincent, and O. Arcizet, Nat. Nanotechnol. 9, 920 (2014).

[37] T. Weiss, A. Kronwald, and F. Marquardt, New J. Phys. 18, 013043 (2016).

[38] L. D. Tóth, N. R. Bernier, A. Nunnenkamp, A. K. Feofanov, and T. J. Kippenberg, Nat. Phys. 13, 787 (2017).

[39] F. Marquardt, J. G. E. Harris, and S. M. Girvin, Phys. Rev. Lett. 96, 103901 (2006).

[40] https://doi.org/10.5281/zenodo.1322025

[41] M. Le Bellac, Quantum Physics (Cambridge University Press, New York, 2012).

[42] A. L. Fetter, J. D. Walecka, and Physics, Quantum Theory of Many-Particle Systems (Dover, New York, 2003).

[43] W.-H. Steeb and Y. Hardy, Int. J. Mod. Phys. C 25, 1450059 (2014).

[44] A. Metelmann and A. A. Clerk, Phys. Rev. X 5, 021025 (2015). 